\title{
Reflections of the changes in patent ductus arteriosus management during the last 10 years
}

\author{
Afif EL-Khuffash, ${ }^{1,2}$ Dany E Weisz, ${ }^{3}$ Patrick J McNamara ${ }^{3,4}$
}

${ }^{1}$ Department of Neonatology, Rotunda Hospital, Dublin, Ireland

${ }^{2}$ Department of Paediatrics, Royal College of Surgeons in Ireland, Dublin, Ireland

${ }^{3}$ Department of Paediatrics, University of Toronto, Toronto, Ontario, Canada ${ }^{4}$ Physiology and Experimental Medicine Program, Hospital for Sick Children Research Institute, Toronto, Ontario, Canada

\section{Correspondence to} Dr Patrick J McNamara, Department of Paediatrics, the Hospital for Sick Children, 555 University Ave, Toronto, Ontario, Canada M5G 1X8; patrick.mcnamara@sickkids.ca

Received 12 January 2016 Revised 30 March 2016 Accepted 6 April 2016 Published Online First 26 April 2016

\section{CrossMark}

To cite: EL-Khuffash A, Weisz DE, McNamara PJ. Arch Dis Child Fetal Neonatal Ed 2016;101: F474-F478.

\section{ABSTRACT}

Despite a large body of scientific evidence on the management of premature infants with a patent ductus arteriosus controversy remains and neonatologists remain challenged for knowing which patient to treat, what is the most optimal timing of treatment and which treatments have a positive impact on both short-term and long-term outcomes. In this review article we discuss the increased body of evidence over the past 10 years, much of which questions the role of treatment and suggests the need to reconsider how haemodynamic significance is adjudicated. In addition, we discuss novel approaches to assessment and diagnosis, and highlight areas for future investigation.

'Since the first report by Powell in 1963 of closure of the patent ductus arteriosus in the preterm infant with respiratory distress syndrome, controversy has existed regarding the optimal management of these infants. ${ }^{1}$ This direct quotation from the late 1970 s is testament to a stark reality, that despite 40 years of investment in prospective research and the conductance of more than 50 randomised controlled trials, neonatologists remain challenged by the decision: 'which patent ductus arteriosus (PDA) should we treat?'. The specific reasons for this state of therapeutic uncertainty are likely to be many; however, the consideration of PDA as a dichotomous variable rather than a physiological continuum is the root cause of the problem. The focus of therapeutic intervention has shifted from an approach to close the PDA in all preterm infants and as soon as possible ${ }^{2}$ to suggestions that the PDA may not need to be closed at all. ${ }^{3}$ The argument proposed against therapeutic intervention is based on the lack of benefit to relevant neonatal outcomes, such as bronchopulmonary dysplasia (BPD) or necrotising enterocolitis (NEC), from randomised trials of PDA closure using non-steroidal anti-inflammatory drug trials. The quality of these trials, some conducted over 30 years ago, is challenged by diagnostic heterogeneity and marked variability in the approach to PDA closure which casts doubt over the reliability of any conclusions drawn from any summative evaluation of these studies in aggregation. A recent overview of all PDA trials conducted to date highlighted the lack of standardisation of the definition of a haemodynamically significant PDA. ${ }^{4}$ The association between surgical ligation of the PDA and adverse neonatal outcomes such as BPD, retinopathy of prematurity (ROP) and neurodevelopmental impairment has re-enforced the concern about ductal closure, ${ }^{5-8}$ although it remains unclear whether this reflects a cause-effect relationship or confounding due to illness severity. The cumulative impact of these studies over the past 10 years has challenged neonatologists to question the aggressiveness of their approach to PDA closure and has led to a secular trend away from treatment in some centres. An increase in mortality and rates of BPD in some centres after cessation of PDA treatment suggests the need for reappraisal of contemporary viewpoints. 9 In 2007, our group suggested the need to consider transductal shunt volume as the end point of interest in determining which patients need treatment and appraising the association of haemodynamic significance to relevant neonatal outcomes. ${ }^{10}$ In this review, we discuss the evolution of the science related to diagnosis and treatment, appraise the arguments for/against treatment and highlight areas for future investigation.

\section{THE PREVIOUS 30 YEARS (1976-2005)}

The approach to management during the latter part of the 20th century focused on the non-selective closure of PDA in all preterm infants shortly after birth. This was achieved by using either surgical or medical means. Prophylactic surgical ligation of PDA within a few days following birth was practised in some centres before the widespread use of medical therapy. The only randomised controlled trial of this practice demonstrated a reduction of severe NEC (RR $0.25,95 \%$ CI (0.08 to 0.83 ), $\mathrm{p}$ value 0.02 , NNT 5), but no reduction in mortality, severe grade intraventricular haemorrhage (IVH), CLD and ROP. ${ }^{11}$ The reduction in NEC probably resulted from the timing of PDA treatment rather than the modality. This is supported by the lack of difference in important outcomes between infants randomised to medical therapy versus surgical ligation as the first line closure strategy. ${ }^{12}$ As a result, and due to the significant morbidity associated with surgical ligation, medical therapy of a PDA became the accepted first line approach to closure. ${ }^{13} 14$

Medical prophylaxis, defined as the use of indomethacin, a cyclo-oxygenase inhibitor, within the first $6-12 \mathrm{~h}$ of life, is associated with a reduction in the incidence of severe IVH. ${ }^{15}$ However, this nonselective approach does not reduce the incidence of other important morbidities such as CLD, NEC and neurodevelopment impairment at 18 months corrected gestational age in extremely low birthweight infants. ${ }^{15}$ The clear short-term benefits, most notably reduction in need for surgical ligation, coupled with a lack of demonstrable harm at long-term follow-up and the known suboptimal reliability of neurodevelopmental testing at 18 months, has led to its continued use in some centres. Studies that have followed infants to school 
age have shown an improvement in neurodevelopment in boys who received indomethacin prophylaxis. ${ }^{16}$ Small, presurfactant era placebo-controlled studies of early indomethacin treatment (prior to day 3) for an asymptomatic PDA reported fewer symptomatic PDAs and reduction in the number of days of oxygen therapy in treated infants, but without any change in mortality or morbidity. ${ }^{217-20}$ Despite the limitations of these studies, the general ethos was that the PDA was an important contributor to neonatal morbidity and despite the lack of clear long-term benefits, and uncertainty regarding when intervention should occur and how, treatment should be given. In some centres, an extreme variation of this approach was practised where the PDA was surgically ligated in all high-risk preterm babies and as soon as possible in the 1st week of life. Recent appraisals of the latter approach, demonstrating improved neonatal outcomes after implementation of a more thoughtful and conservative management plan, lend support to the argument that PDA treatment should not be absolute. ${ }^{21} 22$ It is clear from the appraisal of the literature that the short-term benefits of universal prophylaxis (decreased IVH, symptomatic PDA, surgical ligation, PVL) have not translated into improvement in other neonatal or neurodevelopmental outcomes. In particular, the lack of long-term neurodevelopmental benefit of lowered rates of moderate/severe IVH is somewhat surprising and suggests the relationship is more complex. An additional concern regarding universal prophylaxis is that it exposes infants without a PDA to unnecessary treatment, or may close PDAs in infants with elevated pulmonary pressures resulting in potential harm.

\section{PDA TREATMENT: REFLECTIONS OF THE PAST 10 YEARS} Rationale for a permissive approach to duct closure

The association of PDA with increased neonatal mortality, morbidity and neurodevelopmental impairment in early childhood has been the impetus behind decades of clinical trials aimed at mitigating adverse outcomes through PDA prevention or treatment. However a consistent lack of demonstrable improvement in neonatal and neurodevelopmental outcomes has sparked an existential debate regarding the role of ductal closure therapies in the neonatal intensive care unit. ${ }^{323}$ Trials of pharmacological agents have demonstrated improved PDA closure rates and a decrease in symptomatic PDA and surgical ligation, though they were conducted within a clinical practice paradigm of early diagnosis and treatment. Importantly, few, if any, of these trials reported improved neonatal outcomes such as mortality, BPD, ROP or intraventricular haemorrhage. ${ }^{3}$ While PDA prophylaxis and treatment have not been demonstrated to cause harm, the administration of pharmacological and surgical PDA treatments outside the parameters evaluated in RCTs (ie, beyond the first 2 weeks of life) is common, but of uncertain benefit.

Although PDA is strongly associated with adverse outcomes, the demonstrated lack of benefit of closure strategies has led to scepticism regarding whether PDA treatment may mitigate the development of these morbidities. The lack of improvement in outcomes in medical or surgical trials of PDA treatment may reflect several possible explanations: First, PDA may merely be an intermediate in the pathway between prematurity and adverse outcomes, and there may not be a true causal relationship between a haemodynamically significant PDA and neonatal outcomes; Second, a true causal relationship between a haemodynamically significant PDA and adverse outcomes may exist, but may not be alterable by treatment due to the multiplicity of confounding factors that may coexist during a complex NICU course. This may explain why only trials of prophylaxis ${ }^{24}$ (ie, to prevent PDA entirely) or targeted very early treatment of asymptomatic $\mathrm{PDA}^{25}$ have demonstrated reductions in some morbidities; Third, previous study outcomes may have been diluted by inaccurate assessment of 'haemodynamic significance'; Fourth, ubiquitous cross-over of placebo-assigned infants to the active treatment group effectively relegated most trials to evaluating the efficacy of different timing of treatment (eg, early vs late), and may have obscured the benefits of the treatment itself. As a result, 'no' treatment, or treatment under only exceptional circumstances has never truly been tested in a control arm of any treatment trial.

Taken together, criticisms of trials to date suggest the need for identification of a highly selected group of infants where the PDA is deemed to be actively contributing to haemodynamic compromise and the risk of PDA-related morbidities is high. A very narrow window for PDA treatment to mitigate the effects of prolonged exposure to the PDA in the treatment arm, and a moratorium on open-label treatment in the control arm to test the effects of prolonged exposure to PDA are essential in any future trials.

\section{Redefining the approach to medical treatment}

The causal association between a PDA and respiratory morbidity, severe IVH or death before discharge in a preterm infant remains unproven (see figure 1). Previous studies have suffered from selection bias when attempting to identify infants needing treatment. Studies have included all infants with a PDA regardless of haemodynamic significance; therefore, any beneficial effect of treating a significant PDA may have been masked. The presence of confounders is also an issue. Several morbidities, namely NEC, BPD and sepsis, known to be associated with the presence of PDA, may independently lead to death before discharge and poor neurodevelopmental outcome. These factors may increase the apparent association between a PDA and the outcomes in question.

The question of a causal link between a haemodynamically significant PDA and associated morbidities may only be solved with a randomised trial. Trials of treatment should focus on a well defined population of interest guided by echocardiography assessment of PDA shunt volume or surrogate consequences of it and move beyond treatment based on PDA size or presence alone. This process would ensure accurate identification of the potentially harmful effects of the PDA and facilitate treatment of only the high-risk infants. The intervention arm would include a comprehensive assessment of haemodynamic significance during the first $48 \mathrm{~h}$ of life using echocardiography, with treatment initiated only when certain predefined criteria are met. Those criteria should be able to predict the occurrence of haemodynamic consequences related to a PDA in the short term and predict PDA associated morbidities including BPD and NEC in the longer term. Treatment of a PDA when haemodynamic consequences become overt may be too late to modify outcomes. The control arm should not employ any active measures of ductal closure. As the intervention and control arms are not amenable to blinding, there is a potential for selection and measurement bias. Therefore, unmistakeable outcomes, such as death should be used. Personnel measuring other important outcomes (such as radiologists diagnosing severe IVH, and psychologists assessing neurodevelopmental progress) should be blinded to the group allocation. In addition, a thorough randomisation process should be sought to homogenise the two groups in every other aspect. Some restriction on entry criteria (such as gestation, birth weight and inborn/outborn status) may be necessary. This may make the study more feasible but may compromise its external validity. Although the ease of ascertainment of 
Figure 1 Difficulties in assessing a causal relationship between patent ductus arteriosus (PDA) and outcomes. $\mathrm{BPD}$, bronchopulmonary dysplasia; hsPDA, hemodynamically significant patent ductus arteriosus; NEC, necrotising enterocolitis; PET, preeclampsia; PROM, preterm prolonged rupture of membranes; tnECHO, targeted neonatal echocardiography.
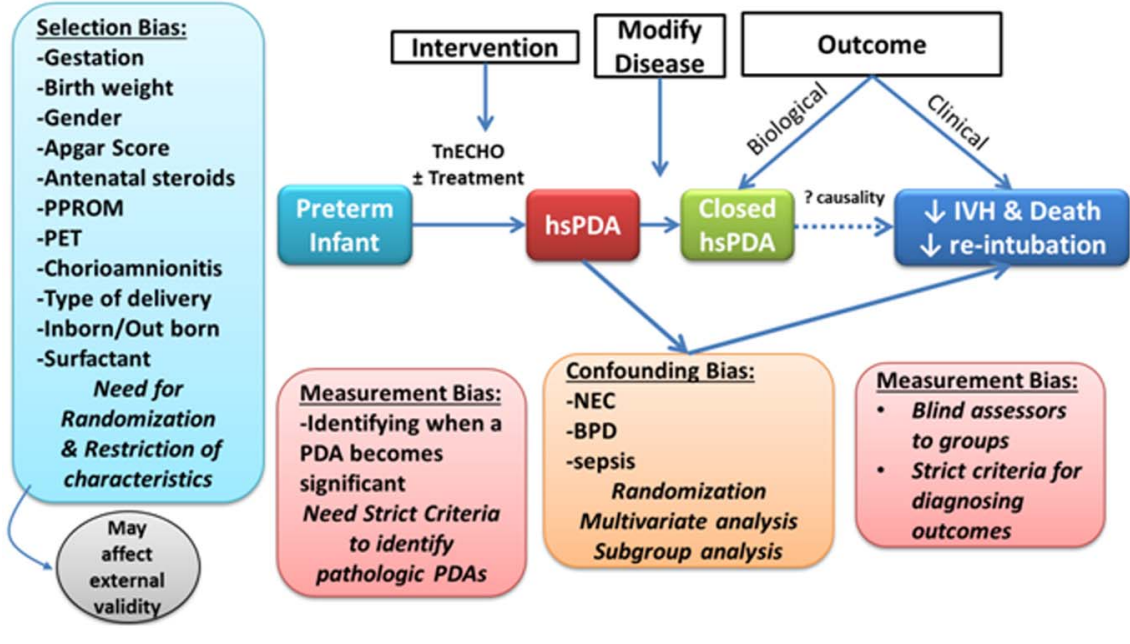

haemodynamic significance increases over the first $48 \mathrm{~h}$, some commentators may be concerned that later intervention may be too late to modify rates of pulmonary and/or intraventricular haemorrhage. Some may also argue that a placebo-controlled trial is unethical due to the increasingly strong association between significant PDAs and important morbidities such as NEC and IVH.

These concerns formed the basis for a prospective observational natural history study to accurately define haemodynamic significance, determine the optimum time of assessment and relate markers of haemodynamic significance to BPD or death before discharge thereby informing future trials of early targeted intervention. ${ }^{26}$ Serial echocardiography was performed at multiple time points in the 1 st week of life in 141 infants under 29 weeks gestation to identify the earliest age and parameters with the best discriminatory ability. Gestational age at birth, PDA diameter and flow velocity, left ventricular output (LVO), and left ventricular (LV) $\mathrm{a}^{\prime}$ wave on day 2 of life were independently associated with CLD/death and were used to devise a PDA severity score (PDAsc). The PDAsc had a range from 0 (low risk) to 13 (high risk). Infants who developed CLD/death had a higher score than those who did not $(7.3$ (1.8) vs $3.8(2.0)$, $\mathrm{p}<0.001)$. A cut-off PDAsc of 5.0 had an area under the curve of $0.92(95 \%$ CI 0.86 to $0.97, \mathrm{p}<0.001)$ for the ability to predict CLD/death. A PDAsc cut-off of 5 has sensitivity and specificity of $92 \%$ and $87 \%$, and positive and negative predictive values of $92 \%$ and $82 \% .^{26}$ These data may be used in future trials to define the population at risk and facilitate a randomised trial of PDA treatment based on echocardiography markers of PDA significance.

\section{Redefining the approach to surgical intervention}

One randomised controlled trial of early prophylactic ligation and several large cohort studies comparing medical and surgical PDA treatment have associated ligation with increased BPD, ROP and neurodevelopmental impairment in early childhood. ${ }^{5-8} 27$ Although prophylactic ligation is now untenable in modern practice and despite a high risk of residual bias in observational studies due to confounding by indication, these findings have been the catalyst for a shift away from surgical PDA ligation over the past decade. ${ }^{28}$ Importantly, the secular trend away from ligation has been contributed to by the emergence of enhanced modalities of non-invasive positive-pressure ventilation that have facilitated the earlier, successful extubation of infants. These methods of non-invasive assisted ventilation are perceived as minimally injurious and have facilitated the prescription of a period of conservative management after failure of pharmacotherapeutic closure, reserving ligation for infants with prolonged persistent significant PDA on clinical and echocardiography grounds. ${ }^{29}$ A delayed selective approach to surgical ligation is supported by a limited number of observational studies that have reported improved BPD and neurodevelopmental impairment compared with early, routine ligation after failure of medical therapy. ${ }^{21} 22$

While the lack of benefit of early ligation was the aggregate finding across study populations of preterm infants with variably significant PDA shunts, it is important to individually consider the small minority of preterm infants who have a persistent very large ductal shunt. In these cases, echocardiographic indices are often in the 'severe' range across all parameters, and infants are typically dependent on mechanical ventilation and may have profound diastolic \pm systolic hypotension and clinical evidence of end-organ hypoperfusion. Early surgical ligation after failure of medical therapy may be indicated for this subgroup of infants. The phenotypical spectrum of the pathophysiological impact of PDA shunting supports the implementation of a staging system of ductal severity to determine the appropriate timing of ligation. ${ }^{30}$ Infants with higher clinical and echocardiographic evidence of physiological impairment related to the ductal shunt should be prioritised for surgical ligation. This is another area warranting an experimental clinical trial to test whether randomising infants with a PDA persisting beyond the 2 nd week of life and categorised as significant based on a staging system would benefit from ligation. However, non-treatment in a control arm where infants are in congestive heart failure and high ventilator requirements may be more ethically challenging than early treatment trials. In addition, as the incidence of infants requiring PDA ligation is in constant decline, a trial of this nature may take several years to complete.

\section{Postligation cardiac syndrome and perioperative management}

Little scientific attention was paid to the postoperative course of neonates undergoing surgical ligation and the approach to intervention prior to 2007 was not evidence-based. PDA ligation results in abrupt haemodynamic changes, with an instantaneous increase in LV afterload and decrease in LV preload, leading to a rapid decline in LV output. Postoperatively, up to half of preterm infants experience a clinically significant gradual decline in LV systolic and diastolic functions. ${ }^{14}$ Signs of a low cardiac output state become apparent $6-12 \mathrm{~h}$ postoperatively and 
comprise a combination of systolic hypotension ( $<3 \mathrm{rd}$ centile) requiring treatment of at least one inotropic agent, accompanied by ventilation and oxygenation failure secondary to LV dysfunction. This clinical deterioration has been termed postligation cardiac syndrome (PLCS). Younger age ( $<28$ days) and weight $(<1000 \mathrm{~g})$ at the time of ligation are preoperative risk factors for PLCS.

A recent study at our centre found that a 1-h postoperative critically low LVO $(<200 \mathrm{~mL} / \mathrm{kg} / \mathrm{min})$, estimated by echocardiography, predicted the development of PLCS in all affected infants. Administering intravenous milrinone prophylaxis to infants with low LVO was associated with a reduction in PLCS from $44 \%$ to $11 \%$, supporting the primary pathological role of increased postoperative LV afterload. Targeted neonatal echocardiography, if available, should be performed at $1 \mathrm{~h}$ postoperatively to estimate LVO. Infants with $\mathrm{LVO}<200 \mathrm{~mL} / \mathrm{kg} / \mathrm{min}$ should receive an intravenous infusion of prophylactic milrinone at a starting dose of $0.33 \mathrm{mcg} / \mathrm{kg} / \mathrm{min}$. In centres without access to timely postoperative echocardiography, the administration of prophylactic intravenous milrinone to infants based on perioperative risk factors may be considered. ${ }^{31}$

\section{Novel approaches to PDA closure}

Concern regarding the long-term impact of PDA ligation and the relatively high rate of side effects associated with nonsteroidal anti-inflammatory drug use in preterm infants has prompted the exploration of novel medical alternatives. Recent observational studies suggest that paracetamol may have a role in PDA closure in infants who are resistant to conventional treatment or those with contraindications to conventional medical therapy. ${ }^{32-34}$ Studies of both oral and intravenous paracetamol have demonstrated similar efficacy to ibuprofen in the early treatment of PDA in preterm infants, though trials have enrolled relatively mature infants. ${ }^{35-37}$ In addition, paracetamol may have a role in the late closure of infants who are candidates for surgical ligation. ${ }^{38}$ However, more studies are needed in a larger cohort to further study its efficacy and safety.

\section{THE NEXT 10 YEARS}

Although there is controversy regarding the optimal approach to PDA management, there has been much scientific advancement in the last 10 years. There is general recognition that the role of the ductus arteriosus varies from benefit to harm, according to the ambient pathophysiological conditions. This has led to a secular trend away from broad non-discriminatory approaches to treatment, with little recognition of the physiological impact of the ductal shunt. While the lack of long-term benefits of pharmacological PDA closure lends merit to the argument for a permissive approach, it is important to recognise that this may not be applicable in all clinical situations, and that physiological strategies to limit shunt volume are oftentimes used in lieu of pharmacological agents. Targeted neonatal echocardiography allows more thoughtful selection of patients, on the basis of surrogate indices of shunt volume, although the long-term benefits of treating a more refined population remain unknown. Although this approach increases the likelihood of identification of the highest-risk patients, who theoretically are most likely to benefit from intervention, it is limited by time; specifically, it may take several days for the shunt volume to be measureable during which interval damage may have ensued. In particular, the prevention of IVH or pulmonary haemorrhage is likely to be impacted by delayed intervention. There remain important gaps in scientific knowledge that need to be addressed. These include investigating the impact of compromised systemic blood flow on organ metabolism and functionality, relative contribution of augmented pulmonary blood flow on lung vascular development, and the effect of both targeted prophylaxis and treatment of patients with established shunts on relevant neonatal morbidity. Finally, future research should interrogate the relationship of prolonged patency of a high volume shunt and PDA closure strategies on brain function and long-term neurodevelopmental outcome.

Contributors $\mathrm{AE}-\mathrm{K}$ and $\mathrm{DEW}$ were responsible for contributing to the review article design, writing a subsection of the first draft of this manuscript, editing this subsection after feedback and reviewing the final draft prior to submission. PJM was responsible for overseeing the design and writing of this review article, writing a subsection of the first draft of this manuscript, editing this subsection and finalising the final draft prior to submission.

Competing interests None declared.

Provenance and peer review Commissioned; externally peer reviewed.

\section{REFERENCES}

1 Merritt TA, DiSessa TG, Feldman BH, et al. Closure of the patent ductus arteriosus with ligation and indomethacin: a consecutive experience. J Pediatr 1978;93:639-46.

2 Mahony L, Carnero V, Brett C, et al. Prophylactic indomethacin therapy for patent ductus arteriosus in very-low-birth-weight infants. $N$ Engl J Med 1982;306:506-10.

3 Benitz WE. Treatment of persistent patent ductus arteriosus in preterm infants: time to accept the null hypothesis? J Perinatol 2010;30:241-52.

4 Zonnenberg I, de Waal K. The definition of a haemodynamic significant duct in randomized controlled trials: a systematic literature review. Acta Paediatr 2012;101:247-51

5 Kabra NS, Schmidt B, Roberts RS, et al. Neurosensory impairment after surgical closure of patent ductus arteriosus in extremely low birth weight infants: results from the Trial of Indomethacin Prophylaxis in Preterms. J Pediatr 2007;150:229-34, 234.e1.

6 Madan JC, Kendrick D, Hagadorn Jl, et al. Patent ductus arteriosus therapy: impact on neonatal and 18-month outcome. Pediatrics 2009;123:674-81.

7 Mirea L, Sankaran K, Seshia M, et al. Treatment of patent ductus arteriosus and neonatal mortality/morbidities: adjustment for treatment selection bias. J Pediatr 2012;161:689-94.

8 Janz-Robinson EM, Badawi N, Walker K, et al. Neonatal Intensive Care Units Network. Neurodevelopmental outcomes of premature infants treated for patent ductus arteriosus: a population-based cohort study. J Pediatr 2015; 167:1025-32.

9 Brooks JM, Travadi JN, Patole SK, et al. Is surgical ligation of patent ductus arteriosus necessary? The Western Australian experience of conservative management. Arch Dis Child Fetal Neonatal Ed 2005;90:F235-F9.

10 McNamara PJ, Sehgal A. Towards rational management of the patent ductus arteriosus: the need for disease staging. Arch Dis Child Fetal Neonatal Ed 2007;92: F424-F7.

11 Cassady G, Crouse DT, Kirklin JW, et al. A randomized, controlled trial of very early prophylactic ligation of the ductus arteriosus in babies who weighed $1000 \mathrm{~g}$ or less at birth. N Engl J Med 1989:320:1511-16.

12 Gersony WM, Peckham GJ, Ellison RC, et al. Effects of indomethacin in premature infants with patent ductus arteriosus: results of a national collaborative study. J Pediatr 1983;102:895-906.

13 Mikhail M, Lee W, Toews W, et al. Surgical and medical experience with 734 premature infants with patient ductus arteriosus. I Thorac Cardiovasc Surg 1982;83:349-57.

14 El-Khuffash AF, Jain A, McNamara PJ. Ligation of the patent ductus arteriosus in preterm infants: understanding the physiology. J Pediatr 2013;162:1100-6.

15 Schmidt B, Asztalos EV, Roberts RS, et al. Impact of bronchopulmonary dysplasia, brain injury, and severe retinopathy on the outcome of extremely low-birth-weight infants at 18 months: results from the trial of indomethacin prophylaxis in preterms. JAMA 2003;289:1124-9.

16 Ment LR, Vohr BR, Makuch RW, et al. Prevention of intraventricular hemorrhage by indomethacin in male preterm infants. J Pediatr 2004;145:832-4.

17 Kääpä P, Lanning P, Koivisto M. Early closure of patent ductus arteriosus with indomethacin in preterm infants with idiopathic respiratory distress syndrome. Acta Paediatr Scand 1983;72:179-84.

18 Hammerman C, Strates E, Valaitis S. The silent ductus: its precursors and its aftermath. Pediatr Cardiol 1986;7:121-7.

19 Weesner KM, Dillard RG, Boyle RJ, et al. Prophylactic treatment of asymptomatic patent ductus arteriosus in premature infants with respiratory distress syndrome. South Med J 1987;80:706-8 
20 Cooke L, Steer P, Woodgate P. Indomethacin for asymptomatic patent ductus arteriosus in preterm infants. Cochrane Database Syst Rev 2003;(2):CD003745.

21 Jhaveri N, Moon-Grady A, Clyman RI. Early surgical ligation versus a conservative approach for management of patent ductus arteriosus that fails to close after indomethacin treatment. J Pediatr 2010;157:381-7.

22 Wickremasinghe AC, Rogers EE, Piecuch RE, et al. Neurodevelopmental outcomes following two different treatment approaches (early ligation and selective ligation) for patent ductus arteriosus. J Pediatr 2012;161:1065-72.

23 Clyman RI, Saha S, Jobe A, et al. Indomethacin Prophylaxis for Preterm Infants: The Impact of 2 Multicentered Randomized Controlled Trials on Clinical Practice. J Pediatr 2007; 150:46-50.

24 Fowlie PW, Davis PG, McGuire W. Prophylactic intravenous indomethacin for preventing mortality and morbidity in preterm infants. Cochrane Database Syst Rev 2010;(7):CD000174.

25 Kluckow $M$, Jeffery $M$, Gill $A$, et al. A randomised placebo-controlled trial of early treatment of the patent ductus arteriosus. Arch Dis Child Fetal Neonatal Ed 2014;99:F99-F104.

26 El-Khuffash A, James AT, Corcoran JD, et al. A patent ductus arteriosus severity score predicts chronic lung disease or death before discharge. J Pediatr 2015;167:1354-61.

27 Clyman R, Cassady G, Kirklin JK, et al. The role of patent ductus arteriosus ligation in bronchopulmonary dysplasia: reexamining a randomized controlled trial. J Pediatr 2009;154:873-6.

28 Shah P, Mirea L, Weisz D, et al. Trends in patent ductus arteriosus management and association with neonatal mortality and morbidities among very preterm infants in Canada. E-PAS 2014:2850.8.

29 Clyman RI, Couto J, Murphy GM. Patent ductus arteriosus: are current neonatal treatment options better or worse than no treatment at all? Semin Perinatol 2012;36:123-9.
30 Resende MHF, More K, Nicholls DB, et al. The impact of a dedicated PDA ligation triaging and management system: a single centre experience. Arch Dis Child Fetal Neonatal Ed 2014;99(Suppl 1):A46-7.

31 Jain A, Sahni M, El-Khuffash A, et al. Use of targeted neonatal echocardiography to prevent postoperative cardiorespiratory instability after patent ductus arteriosus ligation. J Pediatr 2012;160:584-9.

32 Hammerman C, Bin-Nun A, Markovitch E, et al. Ductal closure with paracetamol: a surprising new approach to patent ductus arteriosus treatment. Pediatrics 2011;128: e1618-e21.

33 Alan S, Kahvecioglu D, Erdeve O, et al. Is Paracetamol a useful treatment for ibuprofen-resistant patent ductus arteriosus? Concerning the article by M.Y. Oncel et al: intravenous paracetamol treatment in the management of patent ductus arteriosus in extremely low birth weight infants [Neonatology 2013;103:166-169]. Neonatology 2013;104:168-9.

34 El-Khuffash A, Jain A, Corcoran D, et al. Efficacy of paracetamol on patent ductus arteriosus closure may be dose dependent: evidence from human and murine studies. Pediatr Res 2014;76:238-44.

35 Dang D, Wang D, Zhang C, et al. Comparison of oral paracetamol versus ibuprofen in premature infants with patent ductus arteriosus: a randomized controlled trial. PLOS ONE 2013;8:e77888.

36 Oncel MY, Yurttutan S, Erdeve 0, et al. Oral paracetamol versus oral ibuprofen in the management of patent ductus arteriosus in preterm infants: a randomized controlled trial. J Pediatr 2014;164:510-14.e1.

37 Oncel MY, Yurttutan S, Degirmencioglu H, et al. Intravenous paracetamol treatment in the management of patent ductus arteriosus in extremely low birth weight infants. Neonatology 2013;103:166-9.

38 EL-Khuffash A, James AT, Cleary A, et al. Late medical therapy of patent ductus arteriosus using intravenous paracetamol. Arch Dis Child Fetal Neonatal Ed 2015;100: F253-F6. 